\title{
Interferometer Response to Scalar Gravitational Waves
}

\author{
C. Corda ${ }^{1}$, S. A. Ali ${ }^{2}$ and C. Cafaro ${ }^{3}$
}

October 24, 2018

${ }^{1}$ Institute for Basic Research, P. O. Box 1577, Palm Harbor, FL 34682, USA and Associazione Galileo Galilei, Via Pier Cironi 16 - 59100 Prato, ITALY

${ }^{2}$ Department of Physics, State University of New York at Albany, 1400 Washington Avenue, Albany, NY 12222, USA and Department of Arts and Sciences, Albany College of Pharmacy and Health Sciences, Albany, NY 12208, USA

${ }^{3}$ Dipartimento di Fisica, Università di Camerino, Via Madonna delle Carceri, 9 - Camerino, ITALY

E-mail addresses: ${ }^{1}$ cordac.galilei@gmail.com; ${ }^{2}$ alis@alum.rpi.edu;

${ }^{3}$ carlocafaro2000@yahoo.it

\begin{abstract}
It was recently suggested that the magnetic component of Gravitational Waves (GWs) is relevant in the evaluation of frequency response functions of gravitational interferometers. In this paper we extend the analysis to the magnetic component of the scalar mode of GWs which arise from scalar-tensor gravity theory. In the low-frequency approximation, the response function of ground-based interferometers is calculated. The angular dependence of the electric and magnetic contributions to the response function is discussed. Finally, for an arbitrary frequency range, the proper distance between two test masses is calculated and its usefulness in the high-frequency limit for space-based interferometers is briefly considered.
\end{abstract}


Keywords: Scalar Gravitational Waves; interferometers; magnetic components.

PACS numbers: $04.80 . \mathrm{Nn}, 04.80 .-\mathrm{y}, 04.25 . \mathrm{Nx}$

\section{Introduction}

The study of the so-called magnetic component of Gravitational Waves (GWs) is an active field of research. In a series of recent works, the significance of these magnetic components to the total frequency response function of omnidirectional gravitational wave interferometers was emphasized [1]-4. There are currently a number of gravitational wave detectors at various stages of development worldwide. While several such projects are in their design phase, others like the VIRGO detector (Cascina, Italy [5, 6]) are already under construction. Yet other projects, such as the GEO 600 (Hannover, Germany [7, 8]) the two LIGO detectors (Washington and Louisiana, USA [9, 10]) and the TAMA 300 detector (Tokyo, Japan [11, 12]) are functional and already taking scientific data. In view of such enormous international effort, it is prudent to carry out a thorough analysis of the expected frequency response associated with gravitational waves predicted by General Relativity and its various competing theoretical models of which scalar-tensor gravity is one. Indeed, GW detectors will prove invaluable in the confirmation (or contradiction) of the physical consistency of the various theories of gravity [13]-22]. Baskaran and Grishchuk have recently discussed the existence and relevance of the so-called magnetic components of GWs, which must be taken into account in the context of the total response functions (angular patterns) of interferometers for GWs propagating from arbitrary directions [3]. In [4, more detailed angular and frequency dependence of the magnetic contribution to Interferometer Response Functions was given with specific application to the parameters of the LIGO and VIRGO interferometers.

In this paper the analysis is extended to the magnetic component of the scalar mode of gravitational waves which arise from scalar-tensor theories of gravity $14,15,16,19,21,22$. The angular dependence of the response function of interferometers for this magnetic component is given in the approximation of wavelength much larger than the linear dimensions of the interferometer. The results of this paper generalize the works of $[3,4$, where it was shown that the electric and magnetic contributions are unambiguous in the long-wavelength approximation for ordinary GWs arising from General Relativity. In the high frequency regime, the division into electric and magnetic components becomes ambiguous. For this reason, in order to calculate the response of a $\mathrm{GW}$ interferometer in this regime, we shall use the exact (i.e., without low-frequency approximation) expressions for distance measurements.

The paper is organized as follows. In Section 2, a review of GWs from scalartensor theories of gravity is presented. An analysis of GWs arising from scalartensor gravity theory in the frame of a local observer is implemented in Section 3. 
In Section 4, we obtain the distance function between the two test particles of the interferometer by integrating the geodesic deviation equation. The variation of distances between test masses and the response of interferometers are considered in Section 5. In Section 6, we obtain the interferometer test mass distance function in absence of low-frequency approximation. It is then shown that the electric and magnetic contributions of the distance function arise from the series expansion of this exact result. Our conclusions are presented in Section 7.

\section{Scalar gravitational waves from scalar-tensor theories of gravity}

If the gravitational Lagrangian is nonlinear in the curvature invariants, the corresponding Einstein field equations have an order higher than second [14, 15, 16, 19, 21, 22. For this reason such theories are often called higher-order gravitational theories. In the most general case, such higher order theories arise from an action of form

$$
S=\int d^{4} x \sqrt{-g}\left[F\left(R, \square R, \square^{2} R, \square^{k} R, \phi\right)-\frac{\epsilon}{2} g^{\mu \nu} \phi_{; \mu} \phi_{; \nu}+\mathcal{L}_{m}\right],
$$

where $F$ is an unspecified function of curvature invariants and a scalar field $\phi, D_{\mu} \equiv \partial_{\mu}+\Gamma_{\mu}$ is the covariant derivative with respect to the Christoffel connection coefficients and $\square \equiv g^{\mu \nu} \partial_{\mu} \partial_{\nu}$ is the d'Alembertian operator. The semicolon in $\phi_{; \mu}$ represents $D_{\mu}$-differentiation. The term $\mathcal{L}_{m}$ is the minimally coupled ordinary matter contribution.

In scalar-tensor theories of gravity, both the metric tensor $g_{\mu \nu}$ and a fundamental scalar field $\phi$ are involved [14, 19. The action of scalar-tensor gravity theory can be recovered from (1) with the choice

$$
F(R, \phi)=f(\phi) R-V(\phi) \text { and } \quad \epsilon=-1 .
$$

Note that in this article we work with geometrized units $G=1, c=1$ and

$\hbar=1$. Considering the choice (2), the most general action of scalar-tensor gravity theory in four dimensions is given by

$$
S=\int d^{4} x \sqrt{-g}\left[f(\phi) R+\frac{1}{2} g^{\mu \nu} \phi_{; \mu} \phi_{; \nu}-V(\phi)+\mathcal{L}_{m}\right]
$$

By choosing

$$
\varphi=f(\phi), \quad \omega(\varphi)=\frac{f(\phi)}{2 f^{\prime}(\phi)}, \quad W(\varphi)=V(\phi(\varphi)),
$$

eq. (3) reduces to

$$
S=\int d^{4} x \sqrt{-g}\left[\varphi R-\frac{\omega(\varphi)}{\varphi} g^{\mu \nu} \varphi_{; \mu} \varphi_{; \nu}-W(\varphi)+\mathcal{L}_{m}\right]
$$


Action (51) represents a generalization of Brans-Dicke theory [22]. Variation of action (5) with respect to $g_{\mu \nu}$ and $\varphi$ results in the Einstein-like equation

$G_{\mu \nu}=-\frac{4 \pi \tilde{G}}{\varphi} T_{\mu \nu}^{(m)}+\frac{\omega(\varphi)}{\varphi^{2}}\left(\varphi_{; \mu} \varphi_{; \nu}-\frac{1}{2} g_{\mu \nu} g^{\alpha \beta} \varphi_{; \alpha} \varphi_{; \beta}\right)+\frac{1}{\varphi}\left(\varphi_{; \mu \nu}-g_{\mu \nu} \square \varphi\right)+\frac{1}{2 \varphi} g_{\mu \nu} W(\varphi)$

and Klein-Gordon equation

$$
\square \varphi=\frac{1}{2 \omega(\varphi)+3}\left(-4 \pi \tilde{G} T^{(m)}+2 W(\varphi)+\varphi W^{\prime}(\varphi)+\frac{d \omega(\varphi)}{d \varphi} g^{\mu \nu} \varphi_{; \mu} \varphi_{; \nu}\right.
$$

respectively. The quantity $T_{\mu \nu}^{(m)}$ appearing in (6) is the ordinary stress-energy tensor of matter while $\tilde{G}$ is a dimensional, positive definite constant [14, 17, 19. The Newton constant is replaced by the effective coupling

$$
G_{e f f}=-\frac{1}{2 \varphi}
$$

which is, in general, different from $G$. General Relativity is obtained when the scalar field coupling is

$$
\varphi=\text { const. }=-\frac{1}{2} \text {. }
$$

When $\omega=\omega(\varphi)=$ const. in eqs. (6) and (7) the field equations describe the so-called string-dilaton gravity [14, 17, 19]. Since we study gravitational waves in this work, the linearized theory in vacuum $\left(T_{\mu \nu}^{(m)}=0\right)$ with a small perturbation $h_{\mu \nu}$ of the background will be analysed.

It is assumed that the tensorial sector of the background is Minkowskian $\eta_{\mu \nu}$ while the scalar sector is $\varphi=\varphi_{0}$ where $\varphi_{0}$ is assumed to be a minimum of $W$,

$$
W \simeq \frac{1}{2} \alpha \delta \varphi^{2} \Rightarrow W^{\prime} \simeq \alpha \delta \varphi .
$$

For the perturbed background specified by

$$
g_{\mu \nu}=\eta_{\mu \nu}+h_{\mu \nu}, \quad \varphi=\varphi_{0}+\delta \varphi .
$$

the linearized field equations are given by $[14,19,23$

$$
\widetilde{R}_{\mu \nu}-\frac{\widetilde{R}}{2} \eta_{\mu \nu}=-\partial_{\mu} \partial_{\nu} \Phi+\eta_{\mu \nu} \square \Phi, \quad \square \Phi=m^{2} \Phi,
$$

with

$$
\Phi \equiv-\frac{\delta \varphi}{\varphi_{0}}, \quad m^{2} \equiv \frac{\alpha \varphi_{0}}{2 \omega+3}
$$

where $\widetilde{R}_{\mu \nu \rho \sigma}, \widetilde{R}_{\mu \nu}$ and $\widetilde{R}$ are the linearized versions of $R_{\mu \nu \rho \sigma}, R_{\mu \nu}$ and $R$ respectively, and are moreover, computed to first order in $h_{\mu \nu}$ and $\delta \varphi$.

The case in which $\omega=$ const. and $W=0$ in eqs. (6) and (7) has been analysed in [19] in a manner that generalizes the canonical linearization of General Relativity [23]. In particular, the transverse-traceless (TT) gauge has been 
generalized to scalar-tensor gravity obtaining the total perturbation of a gravitational wave propagating in the $z$-direction (in the TT gauge) as,

$$
h_{\mu \nu}(t+z)=A^{+}(t+z) e_{\mu \nu}^{(+)}+A^{\times}(t+z) e_{\mu \nu}^{(\times)}+\Phi(t+z) e_{\mu \nu}^{(s)} .
$$

The term $A^{+}(t+z) e_{\mu \nu}^{(+)}+A^{\times}(t+z) e_{\mu \nu}^{(\times)}$describes the two standard (i.e. tensorial) polarizations of gravitational waves which arises from General Relativity in the TT gauge [23, while the term $\Phi(t+z) e_{\mu \nu}^{(s)}$ is the extension of the TT gauge to the scalar case. For a purely scalar GW the metric perturbation (14) reduces to

$$
h_{\mu \nu}=\Phi e_{\mu \nu}^{(s)},
$$

and the corresponding line element is [19, 21]

$$
d s^{2}=d t^{2}-d z^{2}-(1+\Phi) d x^{2}-(1+\Phi) d y^{2},
$$

with $\Phi=\Phi_{0} e^{i \omega(t+z)}$.

The worldline $x, y, z=$ const. is a timelike geodesic representing the history of a free test mass (see the analogy with tensorial waves in [3, 4, 23]). In other words, in scalar-tensor theories of gravity the scalar field generates a third tensorial polarization for gravitational waves. This is because in equations (32) of [19] three different degrees of freedom are present, in contrast to standard General Relativity where gravitational waves exhibit two degrees of freedom. For details concerning the generation of this third tensorial polarization, see Section 3 of [19].

\section{Analysis in a locally inertial reference frame}

In a laboratory environment on Earth, a coordinate system (frame of local observer) in which space-time is locally flat is typically used [3, 4, 19, 20, 21, 23. In this frame, the distance between any two points is given simply by the difference in their coordinates (in the sense of Newtonian physics). Moreover, in this frame, SGWs manifest themselves by exerting tidal forces on masses (the mirror and the beam-splitter in the case of an interferometer, see Figure 1).

A detailed analysis of the frame of the local observer is given in ref. [23, Section 13.6. Here only the essential features of this frame are emphasized. In particular, the coordinate $x_{0}$ is the proper time of the observer $O$; spatial axes are centred in $O$; in the special case of zero acceleration and zero rotation, the spatial coordinates $x_{j}$ are the proper distances along the axes and the frame of the local observer reduces to a local Lorentz frame. In this case the line element reads

$$
d s^{2}=-\left(d x^{0}\right)^{2}+\delta_{i j} d x^{i} d x^{j}+O\left(x^{2}\right),
$$

where

$$
O\left(x^{2}\right) \equiv \frac{1}{2}\left(\frac{\partial^{2} g_{\mu \nu}}{\partial x^{\sigma} \partial x^{\rho}}\right)_{P_{0}}\left(x^{\sigma}-x_{P_{0}}^{\sigma}\right)\left(x^{\rho}-x_{P_{0}}^{\rho}\right) d x^{\mu} d x^{\nu}
$$




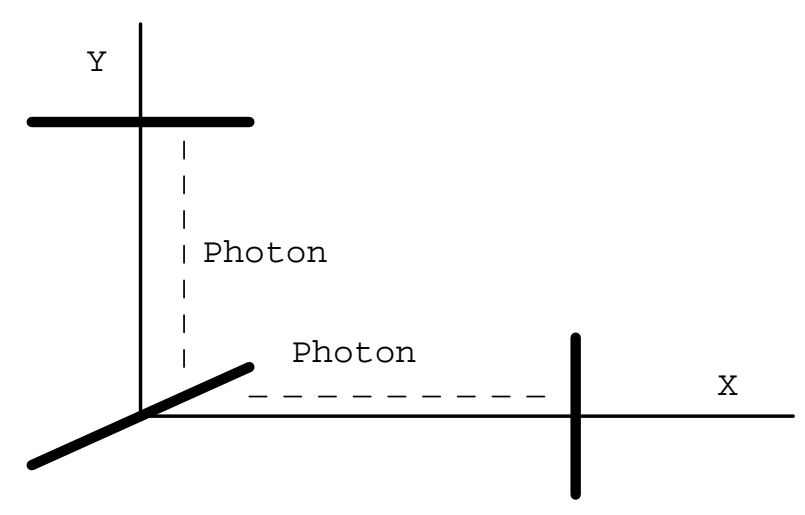

Figure 1: Photons launched from the beam-splitter reflected back by the mirror.

with $P_{0}$ being the point where the Lorentz frame is placed. The effect of SGWs on test masses is described by the equation for geodesic deviation in this frame

$$
\ddot{x}^{\mu}=-\widetilde{R}_{0 \nu 0}^{\mu} x^{\nu}
$$

where $\widetilde{R}_{0 \nu 0}^{\mu}$ are the components of the linearized Riemann tensor [4, 21, 23.

Labelling the coordinates of the TT gauge as $t_{t t}, x_{t t}, y_{t t}, z_{t t}$, the coordinate transformation $x^{\alpha}=x^{\alpha}\left(x_{t t}^{\beta}\right)$ from TT coordinates to the frame of the local observer is

$$
\begin{gathered}
t=t_{t t}+\frac{1}{4}\left(x_{t t}^{2}-y_{t t}^{2}\right) \dot{\Phi} \\
x=x_{t t}+\frac{1}{2} x_{t t} \Phi+\frac{1}{2} x_{t t} z_{t t} \dot{\Phi} \\
y=y_{t t}+\frac{1}{2} y_{t t} \Phi+\frac{1}{2} y_{t t} z_{t t} \dot{\Phi} \\
z=z_{t t}-\frac{1}{4}\left(x_{t t}^{2}-y_{t t}^{2}\right) \dot{\Phi}
\end{gathered}
$$

where $\dot{\Phi} \equiv \frac{\partial \Phi}{\partial t}$ (see the analogy with tensorial waves in [3, 4, 24, 25]). The coefficients of this transformation (components of the metric and its first time derivative) are taken along the central wordline of the local observer [3, 24, 25. The linear and quadratic terms, as powers of $x_{t t}^{\alpha}$, are unambiguously determined by the conditions of the frame of the local observer [3, 4, 24, 25].

Considering a free mass with a timelike geodesic $\left(x=l_{1}, y=l_{2}, z=l_{3}\right)$ [3, 4], equations (20) define the motion of this mass with respect to the introduced frame of the local observer, namely

$$
\begin{gathered}
x(t)=l_{1}+\frac{1}{2} l_{1} \Phi(t)+\frac{1}{2} l_{1} l_{3} \dot{\Phi}(t) \\
y(t)=l_{2}+\frac{1}{2} l_{2} \Phi(t)+\frac{1}{2} l_{2} l_{3} \dot{\Phi}(t) \\
z(t)=l_{3}-\frac{1}{4}\left(l_{1}^{2}-l_{2}^{2}\right) \dot{\Phi}(t) .
\end{gathered}
$$


In absence of GWs the position of the mass is $\vec{x}_{\text {mass }}=\left(l_{1}, l_{2}, l_{3}\right)$. The effect of the SGW is to drive the mass to have oscillations. Thus, in general all three components of motion are present in (21). Neglecting the terms with $\dot{\Phi}$ in (21), the "traditional" equations for the motion of the mass are obtained [19, 21],

$$
\begin{gathered}
x(t)=l_{1}+\frac{1}{2} l_{1} \Phi(t) \\
y(t)=l_{2}+\frac{1}{2} l_{2} \Phi(t) \\
z(t)=l_{3} .
\end{gathered}
$$

Clearly, this is analogous to the electric component of motion in electrodynamics [3, 4, while equations

$$
\begin{gathered}
x(t)=l_{1}+\frac{1}{2} l_{1} l_{3} \dot{\Phi}(t) \\
y(t)=l_{2}+\frac{1}{2} l_{2} l_{3} \dot{\Phi}(t) \\
z(t)=l_{3}-\frac{1}{4}\left(l_{1}^{2}-l_{2}^{2}\right) \dot{\Phi}(t),
\end{gathered}
$$

are analogues of the magnetic component of motion. One might suspect the presence of this magnetic component is a "frame artefact" due to transformation (20). This issue will be addressed in the next Section where it will be shown that (23) is obtained directly from the geodesic deviation equation, verifying that the magnetic components have real physical significance. It is important to observe that the magnetic component become significant when the frequency of the wave increases, but only in the low-frequency regime. This can be understood directly from equations (21). In fact, recalling that $\Phi=\Phi_{0} e^{i \omega(t+z))}$, (21) becomes

$$
\begin{gathered}
x(t)=l_{1}+\frac{1}{2} l_{1} \Phi(t)+\frac{1}{2} l_{1} l_{3} \omega \Phi\left(\omega t-\frac{\pi}{2}\right) \\
y(t)=l_{2}+\frac{1}{2} l_{2} \Phi(t)+\frac{1}{2} l_{2} l_{3} \omega \Phi\left(\omega t-\frac{\pi}{2}\right) \\
z(t)=l_{3}-\frac{1}{4}\left(l_{1}^{2}-l_{2}^{2}\right) \omega \Phi\left(\omega t-\frac{\pi}{2}\right) .
\end{gathered}
$$

Thus, terms with $\dot{\Phi}$ in (21) can be neglected only when the wavelength goes to infinity, while at high-frequencies expansion terms proportional to $\omega l_{i} l_{j}(i, j=$ $1,2,3$ ) corrections in (24) break down.

\section{Equations of motion from geodesic deviation}

In this Section we consider the geodesic deviation extended to second order approximation from which it is shown that the magnetic component of a SGW is not a frame artefact. Consider a two-parameters congruence of geodesics. Let $\gamma_{1}(\tau, r)$ and $\gamma_{2}(\tau, r)$ be two neighboring geodesics with initially-parallel tangent vectors $u^{\alpha}$ and connecting vectors $n^{\beta}$. 
The selector parameter $r$ tells "which" geodesic is being considered, while the affine parameter $\tau$ tells "where" on a given geodesic someone is. Then, the relative acceleration of these two neighboring geodesics is given, at the lowest order of approximation [23, 26, by the Jacobi-Levi-Civita (JLC equation) of geodesic spread,

$$
\frac{D^{2} n^{\delta}}{d \tau^{2}}=R_{\alpha \beta \gamma}^{\delta} u^{\alpha} n^{\beta} u^{\gamma}
$$

where

$$
u^{\alpha}(\tau, r)=\left.\frac{\partial x^{\alpha}(\tau, r)}{\partial \tau}\right|_{r=\text { const }}
$$

is the unit vector tangent to the geodesic $\gamma(\tau, r)$ and

$$
n^{\alpha}(\tau, r)=\left.\frac{\partial x^{\alpha}(\tau, r)}{\partial r}\right|_{\tau=\text { const }} \delta r
$$

is the separation vector between two nearby geodesics. The quantity $R_{\alpha \beta \gamma}^{\delta}$ in (25) is the Riemann curvature tensor defined in the standard way as $R_{\alpha \beta \gamma} \delta=$ $\partial_{\beta} \Gamma_{\alpha \gamma}^{\delta}-\partial_{\gamma} \Gamma_{\alpha \beta}^{\delta}+\Gamma_{\sigma \beta}^{\delta} \Gamma_{\alpha \gamma}^{\sigma}-\Gamma_{\sigma \gamma}^{\delta} \Gamma_{\alpha \beta}^{\sigma}$ and is calculated along the central geodesic. The Christoffel connection coefficients $\Gamma_{\mu \nu}^{\alpha}$ are defined by $\Gamma_{\mu \nu}^{\alpha}=\frac{1}{2} g^{\alpha \sigma}\left(\partial_{\mu} g_{\sigma \nu}+\right.$ $\left.\partial_{\nu} g_{\mu \sigma}-\partial_{\sigma} g_{\mu \nu}\right)$. The operator $\frac{D}{d \mu}$ is the covariant derivative calculated along that line. It is assumed that $r=0$ corresponds to the central geodesic line while a second nearby geodesic corresponds to $r=r_{0}$. To discuss the magnetic component of motion in the field of a SGW we require the geodesic deviation equations be extended to the next order approximation. These equations were obtained in [27], while a modified derivation can be found in [28]. Consider the vector $w^{\alpha}$ defined as

$$
w^{\alpha}=\frac{D n^{\alpha}}{d r}=n_{; \beta}^{\alpha} n^{\beta}=\frac{\partial^{2} x^{\alpha}}{\partial r^{2}}+\Gamma_{\beta \gamma}^{\alpha} u^{\beta} u^{\gamma}
$$

This vector obeys the equations $3,27,28$,

$$
\frac{D^{2} w^{\delta}}{d \tau^{2}}=R_{\alpha \beta \gamma}^{\delta} u^{\alpha} u^{\gamma} w^{\beta}+\left(R_{\alpha \beta \gamma ; \epsilon}^{\delta}-R_{\gamma \epsilon \alpha ; \beta}^{\delta}\right) u^{\alpha} u^{\beta} u^{\gamma} u^{\epsilon}+4 R_{\alpha \beta \gamma}^{\delta} u^{\beta} \frac{D n^{\alpha}}{d \tau} n^{\gamma} .
$$

Defining the vector

$$
N^{\alpha} \equiv r_{0} n^{\alpha}+\frac{1}{2} r_{0}^{2} w^{\alpha}
$$

equations (25) and (28) can be combined to give [3, 27, 28,

$$
\frac{D^{2} N^{\delta}}{d \tau^{2}}=R_{\alpha \beta \gamma}^{\delta} u^{\alpha} u^{\gamma} N^{\beta}+\left(R_{\alpha \beta \gamma ; \epsilon}^{\delta}-R_{\gamma \epsilon \alpha ; \beta}^{\delta}\right) u^{\alpha} u^{\beta} N^{\gamma} N^{\epsilon}+2 R_{\alpha \beta \gamma}^{\delta} u^{\beta} \frac{D N^{\alpha}}{d \tau} N^{\gamma}+O\left(r_{0}^{3}\right) .
$$

It is then possible to write the expansion of $x^{\alpha}\left(\tau, r_{0}\right)$ in terms of $N^{\alpha}$ as 


$$
x^{\alpha}\left(\tau, r_{0}\right)=x^{\alpha}(\tau, 0)+N^{\alpha}-\Gamma_{\beta \gamma}^{\alpha} N^{\beta} N^{\gamma}+O\left(r_{0}^{3}\right) .
$$

This formula shows that in the frame of the local observer (in which the Christoffel connection coefficients $\Gamma_{\beta \gamma}^{\alpha}=0$ along the central geodesic line [23]) the spatial components of $\mathrm{N}$ will directly give the time-dependent position of the nearby test mass. According to (31), these positions include the next-order corrections as compared with solutions to (25). We now specialize to the SGW metric (16), and take into account only linear perturbations in the SGW amplitude. The first test mass is described by the central timelike geodesic $x^{i}(t)=0$, its tangent vector being $u^{\alpha} \equiv(1,0,0,0)$. The second test mass is situated at the unperturbed position $x^{i}(0)=l^{i}$, having zero unperturbed velocity. Assuming that the frame of the local observer is located along the central geodesic, the task is to find the trajectory of the second test mass using the generalized geodesic deviation equation (31). The deviation vector can be written as

$$
N^{i}(t)=l^{i}+\delta l^{i}(t)
$$

where the variation in distance $\delta l^{i}(t)$ is caused by the SGW. In the frame of the local observer one can replace all covariant derivative in (31) by ordinary derivatives [23. In the lowest approximation (31) reduces to (25) and specializes to

$$
\frac{d^{2} \delta l^{i}(t)}{d t^{2}}=-\frac{1}{2} l^{j} \frac{\partial^{2}}{\delta t^{2}} \Phi \delta_{j}^{i}=\frac{1}{2} \omega^{2} l^{j} \Phi e_{j}^{(s) i}
$$

in the field of (16). The relevant solution to (34) coincides exactly with the usual electric part of the motion given by (22). Since we want to identify the magnetic part of the gravitational force arising from a SGW, all terms in (31) must be considered. Since $\frac{D N^{a}}{d \tau}$ is of order $\Phi$, the third term of (31) is of order $\Phi^{2}$ and can be neglected. Computing the derivatives of the curvature tensor and substituting them into (31) specialized in the field of (16), the correct equations of motion read:

$$
\frac{d^{2} \delta l^{i}(t)}{d t^{2}}=\frac{1}{2} \omega^{2} l^{j} \Phi e_{j}^{(s) i}-\frac{1}{2} \omega^{2} l^{k} l^{l}\left(k_{j} \delta^{i j}+\frac{1}{2} k^{i}\right) \delta_{l}^{j} \Phi e_{k j}^{(s)} .
$$

The second term on the right hand side of (35) is responsible for the magnetic component of motion and can be interpreted as the gravitational analogue of the magnetic part of the Lorentz force (see also the analogy for ordinary tensorial waves in [3] and [4]).

\section{Variation of distances between test masses and response of interferometers}

In this Section we are interested in the distance between the central particle, located at the coordinate origin and a particle located on average at some 
position $\left(l_{1}, l_{2}, l_{3}\right)$. This model represents the situation of the beam-splitter and an interferometer [3, 4. In this frame, the Galilean distance - accurate to terms of order $\Phi l$ and $\Phi l^{2} \omega$ while neglecting terms quadratic in $\Phi$ - is given by,

$$
d(t)=\sqrt{x^{2}+y^{2}+z^{2}}+O\left((\Phi l(\omega l))^{2}\right),
$$

Letting

$$
x=l_{1}+\delta x, \quad y=l_{2}+\delta y, \quad z=l_{3}+\delta z,
$$

in (36) we get

$$
d(t)=l+\frac{1}{l}\left(l_{1} \delta x+l_{2} \delta y+l_{3} \delta z\right)
$$

By using the time dependent positions (24), the distance $d(t)$ takes the form (with the required approximation $\omega l \ll 1$ ):

$$
d(t)=l+\frac{1}{2 l}\left(l_{1}^{2}-l_{2}^{2}\right) \Phi(\omega t)-\frac{1}{4 l} \omega l_{3}\left(l_{1}^{2}-l_{2}^{2}\right) \Phi\left(\omega t-\frac{\pi}{2}\right) .
$$

The first correction to $l$ is due to the electric contribution, while the second correction to $l$ is due to the magnetic contribution. According to (24), the magnetic component of motion is present even if the mean position of the second test mass is such that $l_{3}=0$, in perfect analogy with the pure General Relativity case shown in [3, 4].

Now we consider the response of a laser interferometer. To compute the response function of the interferometer to a SGW from arbitrary propagating directions we recall that the arms of the interferometer are in the $\vec{u}$ and $\vec{v}$ directions, while the $x, y, z$ frame is adapted to the propagating SGW. Therefore, performing a spatial rotation of the coordinate system, we obtain

$$
\begin{array}{ccc}
u & = & -x \cos \theta \cos \phi+y \sin \phi+z \sin \theta \cos \phi \\
v & = & -x \cos \theta \sin \phi-y \cos \phi+z \sin \theta \sin \phi \\
w & = & x \sin \theta+z \cos \theta,
\end{array}
$$

or, in terms of the $x, y, z$ frame:

$$
\begin{array}{lcc}
x & = & -u \cos \theta \cos \phi-v \cos \theta \sin \phi+w \sin \theta \\
y & = & u \sin \phi-v \cos \phi \\
z & = & u \sin \theta \cos \phi+v \sin \theta \sin \phi+w \cos \theta
\end{array}
$$

In this way the SGW is propagating from an arbitrary direction $\vec{r}$ to the interferometer (see figure 2). The response function $\delta d(t)$ is defined by

$$
\delta d(t) \equiv d_{u}(t)-d_{v}(t),
$$

where $d_{u}(t)$ and $d_{v}(t)$ are the distances in the $u$ and $v$ direction. Using equations (39), (40), (41) and (42) we obtain 


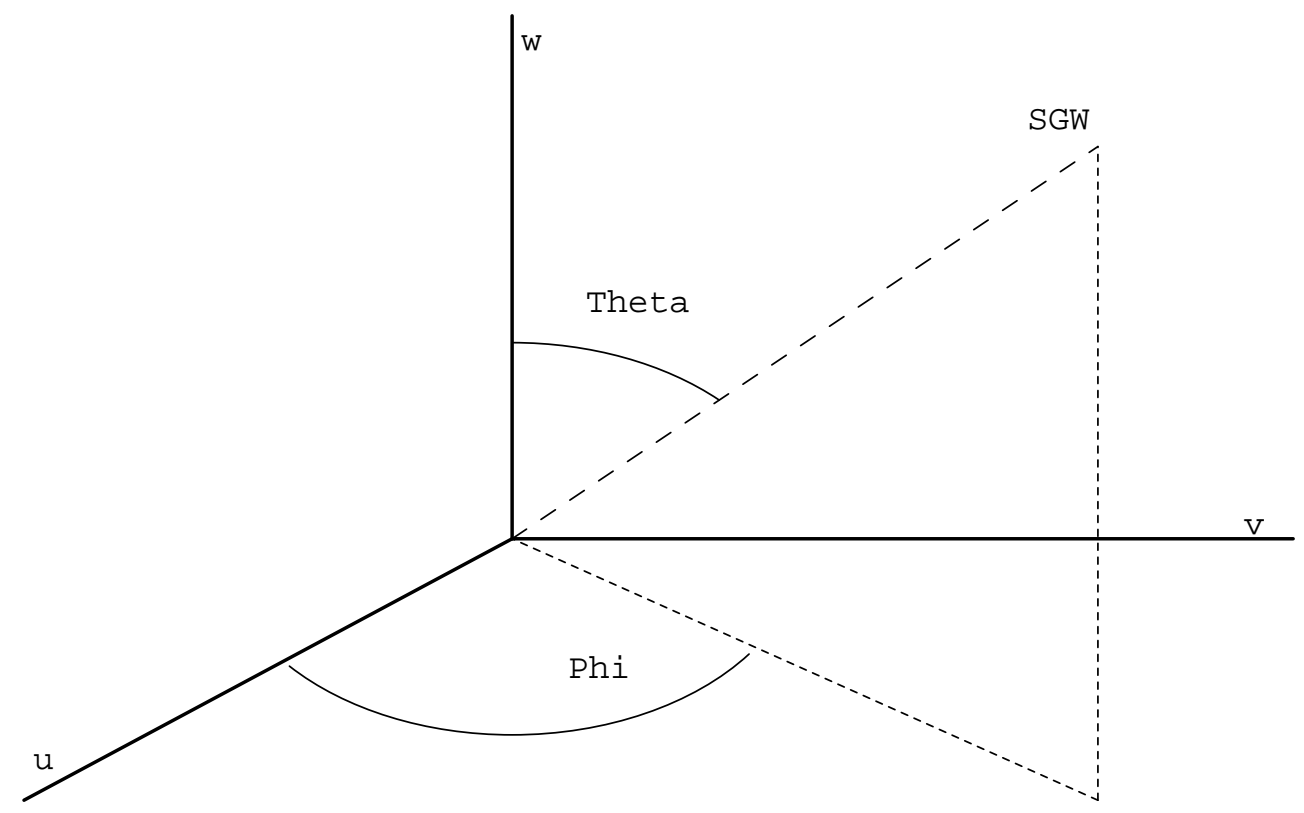

Figure 2: A SGW propagating from an arbitrary direction

$\delta d(t)=-\Phi(t) l \sin ^{2} \theta \cos 2 \phi+\frac{1}{4} \Phi(t) \omega l^{2} \cos \theta\left\{\left[\left(\frac{1+\sin ^{2} \theta}{2}\right)+\sin ^{2} \theta \sin 2 \phi\right](\cos \phi-\sin \phi)\right\}$.

The first term in (43) is due to the electric contribution, while the second term is due to the magnetic contribution. Our response function (43) is more complete than the previously derived expression in [19, 21, 29, since it includes an intrinsic magnetic contribution. The function $\frac{1}{4} \omega l \cos \theta\left[\left(\frac{1+\sin ^{2} \theta}{2}\right)+\right.$ $\left.\sin ^{2} \theta \sin 2 \phi\right)(\cos \phi-\sin \phi)$ ] represents the so-called "angular pattern" [19] of interferometers for the magnetic contibution of SGWs. A plot of the absolute value of this function is shown for the Virgo and LIGO interferometers in Figure 3 and 4 respectively, for a frequency of $f=1000 \mathrm{~Hz}$ in each case.

\section{Variation of distance from the theory of Scalar Gravitational Waves}

It is important to verify that the approximate expression (39) follows directly from the exact definition of distance which arises from the theory of SGWs. For this purpose we make use of the time of flight measurement of a photon travelling from one test mass to another and back. Indeed, such consideration of photon 


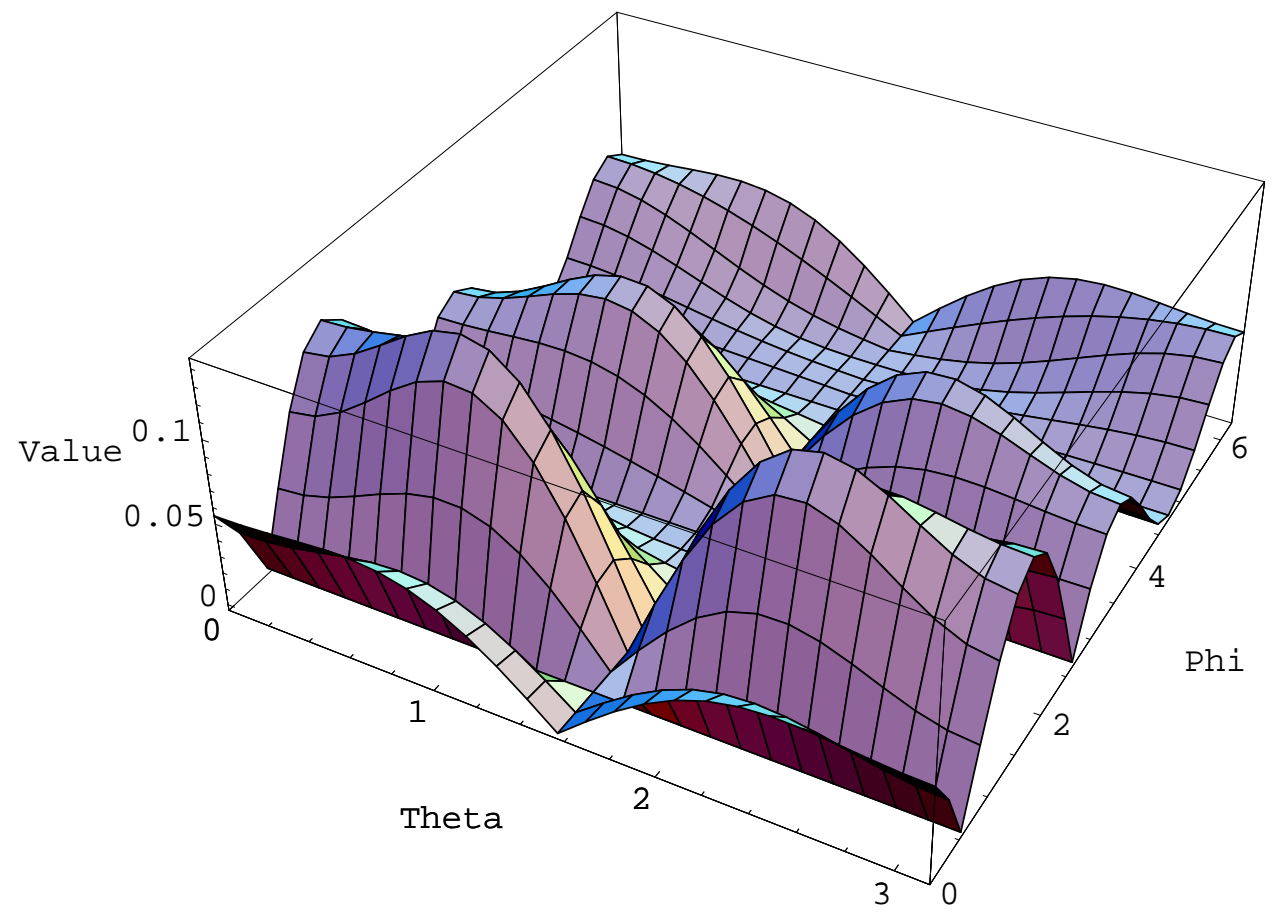

Figure 3: The absolute value of the angular dependence of the total response function of the Virgo interferometer to the magnetic component of a SGW for $f=1000 H z$ 


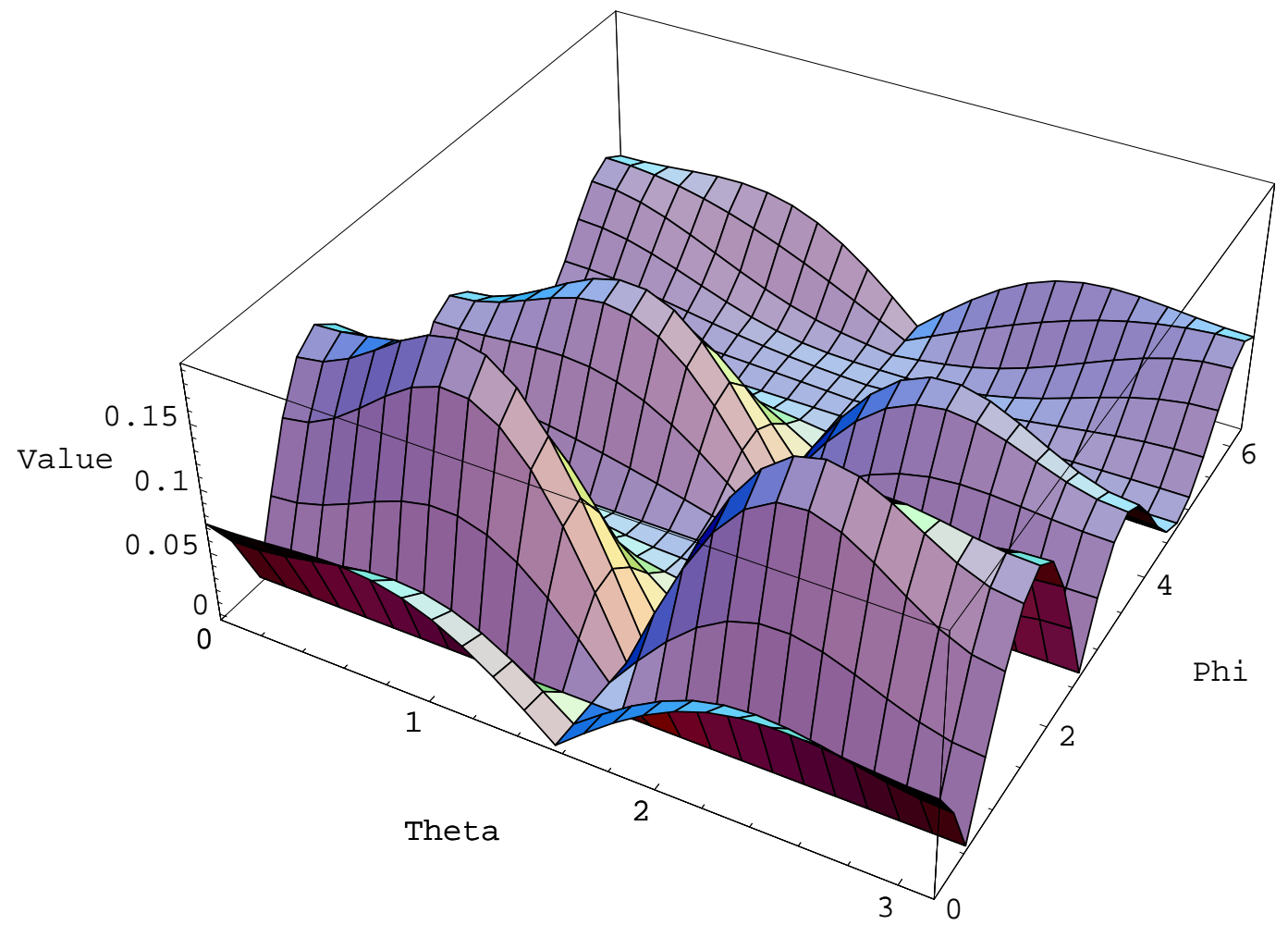

Figure 4: The absolute value of the angular dependence of the total response function of the LIGO interferometer to the magnetic component of a SGW for $f=1000 H z$ 
transit time is an integral part of the more general problem of finding the null geodesics of light in the presence of weak gravitational waves [3, 4, 19, 20, 23, 24, 25]. This definition of distance $d(t)$ is valid independent of the relationship between $l$ and $\omega$ and does not require the introduction of the frame of the local observer. Consider a photon emitted from the beam-splitter of an interferometer at instant $t_{0}$. Furthermore, assume the same photon is reflected by a mirror and returns to the beam-splitter at instant $t_{1}$. The proper distance $d(t)$ between the two test masses at time $t$ is defined as

$$
d(t)=\frac{t_{1}-t_{0}}{2},
$$

where $t=\frac{t_{1}+t_{0}}{2}$ is the mean time between the departure and arrival of the photon. In the field of a SGW, the trajectories of photons are described by the null geodesic $d s^{2}=0$, which from (16), leads to

$$
d t^{2}=(1+\Phi) d x^{2}+(1+\Phi) d y^{2}+d z^{2} .
$$

The unperturbed outgoing photon can be parametrized as 3 ]

$$
t=t_{0}+l \zeta, \quad x=l_{1} \zeta, \quad y=l_{2} \zeta, \quad z=l_{3} \zeta
$$

with $0 \leq \zeta \leq 1$. Thus, according to equation (45) we obtain

$$
d t=l\left[1+\Phi(\zeta) \frac{l_{1}^{2}+l_{2}^{2}}{l^{2}}\right] d \zeta
$$

Integrating both the sides of this equation, we find the time of arrival of the photon at the mirror. In an analogous manner, the unperturbed reflected photon can be parametrized as

$$
t=t_{1}-t_{0}+l \zeta, \quad x=l_{1}-l_{1} \zeta, \quad y=l_{2}-l_{2} \zeta, \quad z=l_{3}-l_{3} \zeta,
$$

where it is $0 \leq \zeta \leq 1$ again. An analogous integration of (47) gives the time of arrival of the photon back at the beam splitter (from the mirror). Combining the two pieces of the transit of the photon from the beam splitter to mirror and back enables one to obtain an exact expression for the distance $d(t)$, namely

$$
d(t)=l+\frac{l_{1}^{2}-l_{2}^{2}}{4 l}\left[\frac{\Phi\left(\omega t+\omega l_{3}\right)-\Phi(\omega t+\omega l)}{\omega\left(l-l_{3}\right)}-\frac{\Phi\left(\omega t+\omega l_{3}\right)-\Phi(\omega t-\omega l)}{\omega\left(l+l_{3}\right)}\right] .
$$

In the low frequency approximation and retaining only the first two terms in the expansion of $\Phi$, (49) reduces to (39). Equation (39) is sufficient only for ground based interferometers, for which the condition $\omega \ll 1 / l$ is in general satisfied. In the case of space-based interferometers for which the above condition is not satisfied in the high-frequency region of the sensitivity band [3, 4], the theory of scalar gravitational waves (49) must be employed. Having shown that formula (39) follows from the exact distance (49) suggests the magnetic contribution to the distance is an universal physical phenomenon which has to be taken into account in the data analysis of GW interferometers. 


\section{Conclusions}

In this paper the motion of a free test particle in the field of a scalar gravitational wave arising from scalar-tensor theories of gravity is considered in detail by solving higher order geodesic deviation equations. The presence and significance of the so-called electric and magnetic contributions to the distance function between interferometer test masses have been shown. The angular dependence of the response function of interferometers due to the magnetic correction to the distance function in the low-frequency approximation was obtained. These results generalize previous works in the literature, where it has been shown that the electric and magnetic contributions are unambiguous in the long-wavelength approximation for ordinary GWs arising from General Relativity. Finally, for arbitrary frequency range, the proper distance between the two interferometer test masses is calculated and its usefulness in the high-frequency limit for space based interferometers is briefly considered.

The response function (i.e., variation of distance) which has been obtained is more complete than the previous derived expressions in the literature (see [16, 19, 21, 30] for example), because such a response function includes the magnetic contribution. In the high-frequency regime the division between electric and magnetic components becomes ambiguous, thus requiring use of the theory of scalar gravitational waves without (low-frequency) approximation. The exact expression for the response function was derived from which it was verified that the electric and magnetic corrections follow from a series expansion of the exact result. We also emphasize that the presence of the higher order terms in the frequency dependent response function become crucial for space-based interferometers, since the (low-frequency) condition $\omega \ll 1 / l$ is not satisfied in the high-frequency portion of the sensitivity band of the interferometer.

The response function contains contributions from two terms, namely the electric and magnetic, both of which have been explicitly derived and explained.

The detector response function of laser gravitational wave interferometers with arms of finite-size (few kilometers length) is commonly described in terms of the long-wavelength approximation. In [3], it was estimated that errors arising from the description of the detector response in terms of such approximation can be as large as $10 \%$. In order to improve the estimation of parameters of gravitational waves it was suggested that the linear-frequency approximation be used, where the response function becomes the sum of two terms, namely the electric one (frequency-independent term) and the magnetic one (linear in the frequency). A detailed analysis of such theoretical estimates as well as new estimates of high-frequency corrections to the detector response appear in [31. In that work, it was argued that errors arising from the description of the detector response in the low-frequency approximation for standard General Relativity, periodic GWs and isotropic stochastic background searches can be as large as $1-2 \%$ at $f=1.2 k H z$. Moreover, the exact formula for the detector response is recommended whenever the accuracy of the detector response is important, especially in high-frequency regimes $(f=37.5 \mathrm{kHz}$ for LIGO interferometers).

Our findings suggest the magnetic component of SGWs is an universal physi- 
cal phenomenon that must be taken into account so as not to discard potentially relevant gravitational wave interferometer data.

\section{Acknowledgements}

We thank Salvatore Capozziello, Mauro Francaviglia, Maria Felicia De Laurentis and Giancarlo Cella for helpful advice during this work. We thank an anonymous Referee for useful comments which led to the improvement of this article. Thanks is also extended to the European Gravitational Observatory (EGO) consortium and to the Associazione Scientifica Galileo Galilei for use of their computing facilities.

\section{References}

[1] C. Corda, Proceedings of the XLIInd Rencontres de Moriond, Gravitational Waves and Experimental Gravity, p. 95, Ed. J. Dumarchez and J. T. Tran, Than Van, THE GIOI Publishers (2007)

[2] C. Corda, Int. J. Mod. Phys. D 16, 9, 1497-1517 (2007)

[3] D. Baskaran and L. P. Grishchuk, Class. Quant. Grav. 21 4041-4061 (2004)

[4] C. Corda, Int. J. Mod. Phys. A 22, n. 13, 2361 -2381 (2007)

[5] C. Corda, Astropart. Phys. 27, No 6, 539-549 (2007)

[6] F. Acernese et al. (the Virgo Collaboration), Class. Quant. Grav. 238 S63-S69 (2006)

[7] F. Acernese et al. (the Virgo Collaboration), Class. Quant. Grav. 238 S63-S69 (2006)

[8] B. Willke et al., Class. Quant. Grav. 23 8S207-S214 (2006)

[9] D. Sigg (the LIGO Scienti...c Collaboration); online at: www.ligo.org/pdf_public/P050036.pdf

[10] B. Abbott et al. (the LIGO Scienti...c Collaboration), Phys. Rev. D 72, $042002(2005)$

[11] M. Ando and the TAMA Collaboration, Class. Quant. Grav. 197 1615-1621 (2002)

[12] D. Tatsumi, Y. Tsunesada and the TAMA Collaboration, Class. Quant. Grav. 215 S451-S456 (2004)

[13] K. S. Thorne in 300 years of gravitation p. 330, Ed. S.W. Hawking and W. Israel, Cambridge: Cambridge University Press (1987) 
[14] S. Capozziello, Newtonian Limit of Extended Theories of Gravity in Quantum Gravity Research Trends Ed. A. Reimer, pp. 227-276 Nova Science Publishers Inc., NY (2005); online at: arXiv: gr-qc/0412088 (2004)

[15] R. H. Dicke - Phys. Rev. 125, 2163 (1962)

[16] M. E. Tobar, T. Suzuki and K. Kuroda, Phys. Rev. D 59102002 (1999)

[17] G. Allemandi, M. Francaviglia, M. L. Ruggiero and A. Tartaglia, Gen. Rel. Grav. 3711 (2005)

[18] G. Allemandi, M. Capone, S. Capozziello and M. Francaviglia, Gen. Rel. Grav. 381 (2006)

[19] S. Capozziello and C. Corda, Int. J. Mod. Phys. D 151119 -1150 (2006)

[20] C. Corda, J. Cosmol. Astropart. Phys. JCAP04009 (2007); C. Corda, Astropart. Phys. 28, 2, 247-250 (2007)

[21] M. Maggiore and A. Nicolis, Phys. Rev. D 62024004 (2000)

[22] C. Brans and R.H. Dicke, Phys. Rev. 124, 925 (1961)

[23] C. W. Misner, K. S. Thorne and J. A. Wheeler, "Gravitation" W. H. Feeman and Company (1973)

[24] L. P. Grishchuk, Sov. Phys. Usp. 20319 (1977)

[25] L. P. Grishchuk, Sov. Phys. JETP 39402 (1974)

[26] L. Landau and E. Lifšits, "Teoria dei campi" Editori riuniti edition III (1999)

[27] S. L. Bazansky Ann. Inst. H. Poincaré A 27115 (1977)

[28] R. Kerner, J. W. van Holten and R. Colistete Jr., Class. Quant. Grav. 18 $4725(2001)$

[29] N. Bonasia and M. Gasperini, Phys. Rev. D 71, 104020 (2005)

[30] M. Shibata, K. Nakao and T. Nakamura, Phys. Rev. D 50, 7304 (1994)

[31] M. Rakhmanov, J. D. Romano, J. T. Whelan, Class. Quant. Grav. 25, 18, 184017 (2008) 